\title{
Association of Early Post-Discharge Follow-Up by a Primary Care Physician and 30-Day Rehospitalization Among Older Adults
}

\author{
Terry S. Field, $D S c^{1,2}$, Jessica Ogarek, $M S^{7}$, Lawrence Garber, $M D^{1}$, George Reed, $P h D^{2}$, and \\ Jerry H. Gurwitz, $M D^{1,2}$ \\ 'Meyers Primary Care Institute, A Joint Endeavor of University of Massachusetts Medical School, Reliant Medical Group, and Fallon Community \\ Health Plan, Worcester, MA, USA; ${ }^{2}$ University of Massachusetts Medical School, Worcester, MA, USA.
}

BACKGROUND: Rehospitalizations within 30 days of discharge are responsible for a large portion of healthcare spending. One approach to preventing rehospitalizations is early follow-up, usually defined as an office visit with a primary care physician within 7 days of discharge-an approach that is being incentivized by health plans. However, evidence regarding its effectiveness is limited.

OBJECTIVE: We aimed to determine whether an office visit with a primary care physician within 7 days after discharge is associated with 30-day rehospitalization.

DESIGN: This was an observational study set within a randomized trial.

PARTICIPANTS: The study included patients age 65 and older receiving care from a multi-specialty group practice and discharged from hospital to home between 26 August 2010 and 25 August 2011. To control for confounding, we identified characteristics of patients and hospital stays that are predictive of rehospitalization, and also developed high-dimensional propensity scores. Analyses used Cox proportional hazards models and took into account varying amounts of opportunity time for office visits.

MAIN MEASURES: We looked at 30-day rehospitalizations at any hospital.

KEY RESULTS: Of 3,661 patients discharged to home during the study year, 707 (19.3\%) were rehospitalized within 30 days. Patients receiving an office visit within 7 days numbered 1,808 (49.4\%), and of these, 1,000 $(27.3 \%)$ were with a primary care physician. In models predicting rehospitalization, stratified on deciles of propensity score and controlling for additional confounders, the hazard ratios associated with office visits with a primary care physician within 7 days were 0.98 (95\% CI $0.80,1.21$ ); for visits with any physician, the hazard ratio was HR 1.04, (95\% CI 0.87, 1.25).

CONCLUSIONS: We found no protective effect for office visits within 7 days. Such visits may need to be specifically focused on a range of issues related to the specific reasons why patients are rehospitalized. It is likely that outpatient

Previous Presentation of Manuscript Information Components of the manuscript were presented at the Health Maintenance Organization Research Network (HMORN) 2014 Conference in Phoenix, Arizona on 3 April 2014, and at the American Geriatrics Society 2014 Annual Meeting in Lake Buena Vista, Florida on 17 May 2014.

Received June 3, 2014

Revised August 22, 2014

Accepted November 6, 2014

Published online December 2, 2014 visits will need to be set within comprehensive transition programs.

KEY WORDS: ambulatory care; care transitions; health services research. J Gen Intern Med 30(5):565-71

DOI: $10.1007 / \mathrm{s} 11606-014-3106-4$

(C) Society of General Internal Medicine 2014

\section{BACKGROUND}

For many older patients, the transition between the inpatient and outpatient setting is a period of particularly high risk. ${ }^{1,2}$ Adverse events are common, as are rehospitalizations. ${ }^{3-5}$ High rates of rehospitalization are considered to be a measure of poor quality of care and an opportunity to reduce Medicare spending. Under the Affordable Care Act, the Centers for Medicare and Medicaid Services has implemented financial penalties for hospitals with 30-day rehospitalization rates that are considered excessive. ${ }^{6-8}$ A landmark publication by Jencks et al. in 2009 reported a 30-day rehospitalization rate of $19.6 \%$ for Medicare enrollees in 2003 and 2004, at an estimated cost of $\$ 17.4$ billion. ${ }^{5}$ Systematic reviews have failed to identify any specific strategy or component that has been effective at reducing rehospitalization.

Timely follow-up with the primary care physician has been suggested as an important component of efforts to optimize transitional care during the high-risk peri-discharge period. ${ }^{9,10}$ Current Procedural Terminology (CPT) codes are now available to support "Transitional Care Management Services," and some health plans have begun to provide financial incentives to medical groups that achieve specified benchmarks relating to prompt post-discharge follow-up visits for older patients.

We conducted an observational study to determine whether an office visit with a primary care physician within 7 days after hospital discharge was associated with a reduction in 30-day rehospitalizations among patients age 65 years and older receiving care from a large multispecialty group practice.

\section{METHODS}

\section{Study Setting, Population and Study Design}

This observational study was set within a randomized controlled trial of an electronic health record (EHR)-based 
transitional care intervention for older adults discharged from hospital to home. ${ }^{11}$ For patients randomized to the intervention arm, messages to schedule an office visit within 1 week of discharge were automatically generated and sent to the primary care providers' support staff, unless scheduling information within the EHR indicated that such a visit had already been scheduled. The intervention did not increase the proportion of discharges with an office visit within 7 days. The randomized trial was conducted within a large multispecialty group practice that employs 265 physicians, including 66 primary care providers who care for adult patients in the outpatient setting, and serves primarily middle and working class patients from the surrounding urban and suburban areas. Hospital care is delivered by hospitalists employed by the medical group. The group provides care to approximately 24,000 senior plan members of an associated health plan. The study population was derived from this population of patients.

We studied patients discharged from the primary hospital used by the group practice from 26 August 2010 to 25 August 2011. The primary hospital is a 350-bed urban community hospital affiliated with a medical school, and includes an internal medicine residency program. Eligibility criteria for inclusion in the study were: (1) patient was 65 years or older at the time of discharge; (2) patient was discharged from the primary inpatient facility serving the medical group for a nonpsychiatric condition; (3) there were no plans to enroll patient in hospice upon discharge; and (4) the patient was discharged to the community (not a skilled nursing facility, rehabilitation facility, or long-term care setting). Confirmation that discharges were to the community was based on manual review of the medical records.

The institutional review board of the University of Massachusetts Medical School and the institutional review board of the multispecialty medical group and the health plan approved this study. The original trial within which this observational study was set was registered with ClinicalTrials.gov (NCT00611091). Funding was from the Agency for Health Care Policy and Research, which had no role in the design, conduct or reporting of the study.

\section{Characteristics of Study Subjects}

We characterized discharged patients according to age at the time of discharge, gender, length of stay, whether a surgical procedure was performed during the hospitalization, hospitalizations during the prior year, office visits during the prior year, number of different providers seen during the prior year in the outpatient setting, number of prescriptions filled during the prior year, and the Charlson Comorbidity Index ${ }^{12}$ using relevant ICD-9 codes derived from health plan administrative data.

\section{Office Visits}

Using encounter data from the medical group's EHR, we determined whether discharged patients had an office visit with a primary care physician within the group in the 7-day period following hospital discharge. We had access to complete data from the health plan, and thus were also able to determine whether patients had office visits with specialist physicians during that period.

\section{Outcome Measures}

The primary outcome was rehospitalization within 30 days of discharge from the index hospitalization. We ascertained information related to hospitalizations from both the medical group's EHR and from health plan data, which allowed for determination of rehospitalizations that occurred at any hospital, not just the primary hospital that served patients cared for by the medical group.

\section{Statistical Analysis}

For direct comparisons of baseline characteristics between those who were and were not rehospitalized within 30 days, we used Fischer exact tests for categorical variables and $t$-tests for continuous variables. Reported $p$ values are two-sided. Comparisons included characteristics of patients, their hospital stays and utilization during the year prior to the index hospitalization. Because of the skewed distributions of age, length of stay and number of prior hospitalizations, we analyzed them in categories defined to optimize their bivariate associations with rehospitalization, as displayed in Table 1 . We categorized potentially relevant components of patients' utilization during the preceding year in quartiles including number of office visits, number of different providers seen, and number of different drugs dispensed. We used time-varying Cox proportional hazards models to assess the association of office visits and rehospitalization. The models focused on time from discharge from the index hospitalization until the first rehospitalization date during the 30 days of follow-up. Patients were censored on date of death, health plan disenrollment, or 30 days post-discharge, whichever came first. For patients with multiple hospitalizations during the study period, we included each discharge and followed it for the following 30 days. Potential confounding variables were determined individually for each discharge. Variables with statistically significant associations with rehospitalization were tested in multivariable proportional hazards models to assess their independent association with rehospitalization and their impact on the hazard ratio for office visits within 7 days. Models also controlled for intervention vs. control status in the randomized trial and for having an office visit within 7 days with a specialist physician.

To further explore possible confounding, we developed a high-dimensional propensity score (HDPS) predicting office visits with the primary care physician within 7 days of discharge, including five dimensions of 
Table 1. Baseline Characteristics of Discharges by Status of Office Visit Within 7 Days, Unmatched and Matched on Propensity Score

\begin{tabular}{|c|c|c|c|c|c|c|}
\hline \multirow[t]{4}{*}{ Characteristic } & \multicolumn{3}{|c|}{ Unmatched: $N=3,661$} & \multicolumn{3}{|c|}{ Propensity score matched: $N=1,704$} \\
\hline & \multirow{2}{*}{$\frac{\text { PCP office visit }}{N=1000}$} & \multicolumn{2}{|l|}{ No PCP office visit } & \multirow{2}{*}{$\begin{array}{l}\text { PCP office visit } \\
N=852\end{array}$} & \multicolumn{2}{|l|}{ No PCP office visit } \\
\hline & & $N=\mathbf{2 6 6 1}$ & & & $N=852$ & \\
\hline & $\begin{array}{l}\text { Mean (standard } \\
\text { deviation) }\end{array}$ & $\begin{array}{l}\text { Mean (standard } \\
\text { deviation) }\end{array}$ & & $\begin{array}{l}\text { Mean (standard } \\
\text { deviation) }\end{array}$ & $\begin{array}{l}\text { Mean (standard } \\
\text { deviation) }\end{array}$ & \\
\hline \multirow[t]{2}{*}{ Patient age at the time of discharge } & $79.6(6.9)$ & $78.8(7.3)$ & & $79.5(7.1)$ & $79.8(7.2)$ & \\
\hline & \# $(\%)$ & \# $(\%)$ & $p$ value & $\#(\%)$ & $\#(\%)$ & $p$ value \\
\hline \multicolumn{7}{|l|}{ Categories of patient ages } \\
\hline $75-84$ & $\begin{array}{l}263(26.3) \\
477(47.7)\end{array}$ & $\begin{array}{l}832(31.3) \\
1,216(45.7)\end{array}$ & 0.01 & $\begin{array}{l}231(27.1) \\
401(47.1)\end{array}$ & $\begin{array}{l}229(26.9) \\
388(45.5)\end{array}$ & 0.70 \\
\hline $85+$ & $260(26.0)$ & $613(23.0)$ & & $220(25.8)$ & $235(27.6)$ & \\
\hline Patient gender-female & $510(51.0)$ & $1,411(53.0)$ & 0.27 & $440(51.6)$ & $446(52.3)$ & 0.77 \\
\hline Intervention & $505(50.5)$ & $1,365(51.3)$ & 0.67 & $435(51.1)$ & $446(52.3)$ & 0.59 \\
\hline \multicolumn{7}{|l|}{ Length of stay } \\
\hline 1 night & $275(27.5)$ & $715(26.9)$ & & $248(29.1)$ & $246(28.9)$ & \\
\hline 2 nights & $276(27.6)$ & $652(24.5)$ & 0.02 & $230(27.0)$ & $231(27.1)$ & 0.99 \\
\hline 3-4 nights & $305(30.5)$ & $800(30.1)$ & & $252(29.6)$ & $250(29.3)$ & \\
\hline $5+$ nights & $144(14.4)$ & 494 (18.6) & & $122(14.3)$ & $125(14.7)$ & \\
\hline Surgery during hospitalization & $98(9.8)$ & $636(23.9)$ & $<0.001$ & $85(10.0)$ & $137(16.1)$ & $<0.001$ \\
\hline \multicolumn{7}{|c|}{ Number of office visits during past year } \\
\hline $0-8$ & $267(26.7)$ & $768(28.9)$ & & $237(27.8)$ & $283(33.2)$ & \\
\hline $9-12$ & $228(22.8)$ & $584(21.9)$ & 0.62 & $196(23.0)$ & $180(21.1)$ & 0.12 \\
\hline $13-18$ & $256(25.6)$ & $654(24.6)$ & & $223(26.2)$ & $209(24.5)$ & \\
\hline $19+$ & $249(24.9)$ & $655(24.6)$ & & $196(23.0)$ & $180(21.1)$ & \\
\hline \multicolumn{7}{|l|}{ Hospitalizations during past year } \\
\hline 0 & $539(53.9)$ & $1493(56.1)$ & & $490(57.5)$ & $485(56.9)$ & \\
\hline $1-2$ & $295(29.5)$ & $706(26.5)$ & 0.20 & 235 (27.6) & $241(28.3)$ & 0.95 \\
\hline $3+$ & $166(16.6)$ & $462(17.4)$ & & $127(14.9)$ & $126(14.8)$ & \\
\hline \multicolumn{7}{|l|}{ Charlson Comorbidity Index } \\
\hline 0 & $146(14.6)$ & $374(14.1)$ & & $126(14.8)$ & $136(16.0)$ & \\
\hline 1 & $210(21.0)$ & $536(20.1)$ & & $191(22.4)$ & $192(22.5)$ & \\
\hline 2 & $175(17.5)$ & $543(20.4)$ & 0.43 & $157(18.4)$ & $154(18.1)$ & 0.94 \\
\hline 3 & 177 (17.7) & $435(16.3)$ & & $143(16.8)$ & $151(17.7)$ & \\
\hline 4 & $107(10.7)$ & $302(11.3)$ & & $88(10.3)$ & $81(9.5)$ & \\
\hline
\end{tabular}

PCP primary care physician

data derived from the EHR and health plan data (inpatient diagnoses, inpatient procedures, outpatient diagnoses, outpatient procedures, and drug dispensing) from the 12 months prior to the index admission. The HDPS calculates an algorithm from claims for outpatient and inpatient care to indirectly serve as a proxy for a patient's underlying health status. The process identifies potential covariates separately within each data dimension, selects the most prevalent, categorizes the frequency with which each of these covariates appears in the data for each patient, prioritizes covariates within each dimension by assessing their potential for controlling confounding not conditional on exposure, and selects the final covariates. We used SAS programs included in the Pharmacoepidemiology Toolbox, ${ }^{13}$ developed by the Division of Pharmacoepidemiology and Pharmacoeconomics at Brigham and Women's Hospital, to calculate the HDPS scores. ${ }^{14} \mathrm{C}$-statistics were calculated to assess the fit of the propensity score model in comparison to logistic regression models predicting primary care physician visits within 7 days with the variables retained in the multivariable proportional hazards model predicting rehospitalization. We matched on propensity score using a greedy matching approach, but only $85 \%$ of the group receiving an office visit could be matched. Therefore, we focused our primary analysis on time-varying Cox proportional hazard model ratios stratified by propensity score decile. As a sensitivity analysis, we also performed a matched analysis to identify any major differences in results.

In addition to the primary models assessing the impact of visits with primary care physicians, we performed a secondary series of analyses on the impact of visits with any physician. This series directly paralleled the approach for visits with primary care physicians and used HDPS scores specifically calculated from predictions of visits with any physician.

SAS software version 9.2 for Unix was used for statistical analyses.

\section{RESULTS}

A total of 3,661 discharges of patients age 65 and older from hospital to home were included in the study. During the 30 days following discharge, 707 patients 
(19.3\%) were rehospitalized. Seventy-eight subjects were censored prior to the end of the 30-day followup period, 74 died and four disenrolled from the health plan and were lost to follow-up.

Table 1 compares discharges followed by office visits with a primary care physician within 7 days to those without. Twenty-seven percent of discharges were followed by an office visit. Factors associated with lower probability of an office visit were longer length of hospital stay, surgery during the index hospitalization, and younger age. Table 1 also describes the sample after matching on HDPS. The two groups are very similar, but only 852 patients $(85 \%)$ of the group with an office visit could be matched, reducing the sample and

Table 2 Baseline Characteristics of Discharges by 30-Day Rehospitalization

\begin{tabular}{|c|c|c|c|}
\hline \multirow[t]{4}{*}{ Characteristic } & \multicolumn{3}{|l|}{$N=3,661$} \\
\hline & \multirow{2}{*}{$\begin{array}{l}\text { Rehospitalized } \\
N=707\end{array}$} & \multicolumn{2}{|l|}{$\begin{array}{l}\text { Not } \\
\text { rehospitalized }\end{array}$} \\
\hline & & \multicolumn{2}{|l|}{$N=2,954$} \\
\hline & $\begin{array}{l}\text { Mean } \\
\text { (standard } \\
\text { deviation) }\end{array}$ & \multicolumn{2}{|l|}{$\begin{array}{l}\text { Mean } \\
\text { (standard } \\
\text { deviation) }\end{array}$} \\
\hline \multirow{2}{*}{$\begin{array}{l}\text { Patient age at the } \\
\text { time of discharge }\end{array}$} & $79.7(7.0)$ & $78.9(7.2)$ & \\
\hline & $\#(\%)$ & \# $(\%)$ & $p$ value \\
\hline \multicolumn{4}{|c|}{ Categories of patient ages } \\
\hline $65-74$ & $190(26.9)$ & $905(30.6)$ & \\
\hline $75-84$ & $314(44.4)$ & $1,379(46.7)$ & $<0.01$ \\
\hline $85+$ & $203(28.7)$ & $670(22.7)$ & \\
\hline $\begin{array}{l}\text { Patient gender- } \\
\text { female }\end{array}$ & $333(47.1)$ & $1,588(53.8)$ & $<0.01$ \\
\hline Intervention & $351(49.7)$ & $1,519(51.4)$ & 0.40 \\
\hline \multicolumn{4}{|l|}{ Length of stay } \\
\hline 1 night & $146(20.7)$ & $844(28.6)$ & \\
\hline 2 nights & $165(23.3)$ & $763(25.8)$ & $<0.001$ \\
\hline 3-4 nights & $224(31.7)$ & $881(29.8)$ & \\
\hline $5+$ nights & $172(24.3)$ & $466(15.8)$ & \\
\hline $\begin{array}{l}\text { Surgery during } \\
\text { hospitalization }\end{array}$ & $132(18.7)$ & $602(20.4)$ & 0.31 \\
\hline \multicolumn{4}{|c|}{ Hospitalizations during past year } \\
\hline 0 & $272(38.5)$ & $1,760(59.6)$ & \\
\hline $1-2$ & $212(30.0)$ & $789(26.7)$ & $<0.001$ \\
\hline $3+$ & $223(31.5)$ & $405(13.7)$ & \\
\hline \multicolumn{4}{|c|}{ Office visits during past year } \\
\hline $0-3$ & $163(23.1)$ & $872(29.5)$ & \\
\hline $4-6$ & $124(17.5)$ & $688(23.3)$ & $<0.001$ \\
\hline $7-11$ & $178(25.2)$ & $732(24.8)$ & \\
\hline $12+$ & $242(34.2)$ & $662(22.4)$ & \\
\hline \multicolumn{4}{|c|}{ \# of different providers during past year } \\
\hline $0-2$ & $147(20.8)$ & $781(26.4)$ & \\
\hline $3-5$ & $206(29.1)$ & $1,071(36.3)$ & $<0.001$ \\
\hline $6-7$ & $128(18.1)$ & $511(17.3)$ & \\
\hline $8+$ & $226(32.0)$ & $591(20.0)$ & \\
\hline \multicolumn{4}{|c|}{ \# of different medications dispensed in past year } \\
\hline$<8$ & $136(19.2)$ & $783(26.5)$ & \\
\hline $8-12$ & $137(19.4)$ & $771(26.1)$ & $<0.001$ \\
\hline $13-19$ & $173(24.5)$ & $714(24.2)$ & \\
\hline $20+$ & $261(36.9)$ & $686(23.2)$ & \\
\hline \multicolumn{4}{|c|}{ Charlson Comorbidity Index } \\
\hline 0 & $74(10.5)$ & $446(15.1)$ & \\
\hline 1 & $93(13.2)$ & $653(22.1)$ & \\
\hline 2 & $130(18.4)$ & $588(19.9)$ & $<0.001$ \\
\hline 3 & $125(17.7)$ & $487(16.5)$ & \\
\hline 4 & $106(15.0)$ & $303(10.2)$ & \\
\hline $5+$ & $179(25.3)$ & $477(16.1)$ & \\
\hline
\end{tabular}

potentially making results of matched analyses difficult to interpret.

A number of demographic and utilization variables were associated with rehospitalization (Table 2). Discharges of older patients, those with longer lengths of hospital stay, additional hospitalizations during the previous year, and higher scores on the Charlson Comorbidity Index were more likely to be followed by rehospitalization within 30 days. Each of the patients' prior utilization variables was also associated with rehospitalization. Women and those who received surgery during the index hospitalization were less likely to be rehospitalized.

We compared a logistic regression model predicting an office visit within 7 days that included the confounding factors retained in the Cox proportional hazards model to the model developed for the high-dimensional propensity score. The cstatistic without HDPS was 0.58, while the c-statistic for the HDPS model was 0.77 .

Results of the series of Cox proportional hazards models as provided in Table 3 demonstrate very little association between having an office visit with a primary care physician and rehospitalization. In the basic model that included no potential confounding factors, the hazard ratio associated with having an office visit was 1.04 (95\% confidence interval (CI) $0.86,1.25)$. Addition of the basic set of potential confounders raised the hazard ratio slightly to 1.07 (95\% CI $0.88,1.29)$. The model stratified on deciles of the HDPS lowered the hazard ratio to 0.98 (95\% CI $0.80,1.21)$. The model matched on HDPS produced a similar hazard ratio of 0.96 (95\% CI $0.75,1.24)$.

Table 3 also presents the results of the secondary analysis series assessing the impact of an office visit with any physician on rehospitalization. Forty-six percent of patients had an office visit with any physician within 7 days. Although the unadjusted model indicated

Table 3 Hazard Ratios for Rehospitalization Within 30 Days Associated with Office Visits Within 7 Days with a Primary Care Physician

\begin{tabular}{|c|c|c|}
\hline & PCP office visit & Any office visit \\
\hline & $\begin{array}{l}\text { Hazard ratio (95\% } \\
\text { confidence interval) }\end{array}$ & $\begin{array}{l}\text { Hazard ratio ( } 95 \% \\
\text { confidence interval) }\end{array}$ \\
\hline Model 1: unadjusted & $1.04(0.86,1.25)$ & $1.21(1.03,1.43)$ \\
\hline $\begin{array}{l}\text { Model 2: adjusted for } \\
\text { basic covariates }\end{array}$ & $1.07(0.88,1.29)^{*}$ & $1.11(0.95,1.32)^{\dagger}$ \\
\hline $\begin{array}{l}\text { Model 3: Model } 2 \\
\text { stratified } \\
\text { by HDPS }\end{array}$ & $0.98(0.80,1.24)$ & $1.04(0.87,1.25)$ \\
\hline $\begin{array}{l}\text { Model 4: Model } 2 \\
\text { with matching on } \\
\text { HDPS }\end{array}$ & $0.96(0.75,1.24)$ & $1.05(0.85,1.29)$ \\
\hline
\end{tabular}

*Adjusted for age, gender, intervention status, surgery during hospitalization, length of hospital stay, hospitalizations during the previous year, Charlson Comorbidity Index, and non-primary care physician office visit within 7 days

'Adjusted for age, gender, intervention status, surgery during hospitalization, length of hospital stay, hospitalizations during the previous year, and Charlson Comorbidity Index 
a statistically significant increased risk (hazard ratio $1.21,95 \%$ CI 1.03, 1.43), control for the basic confounders, stratification on deciles of HDPS, and analysis matched with HDPS score reduced the hazard ratio and eliminated statistical significance (HR 1.04, $95 \%$ CI $0.87,1.25)$.

\section{DISCUSSION}

In this study of one year of discharges from hospital to home for older adults treated by a large medical group, $19.3 \%$ were rehospitalized within 30 days. Although $27.3 \%$ had an office visit with a primary care physician within 7 days, we found no protective effect. A range of possible confounders associated with both rehospitalization and office visits might explain the lack of effect. However, even control for high-dimensional propensity scores did not have a major impact on these results. The results of a parallel, secondary series of analyses assessing the impact of having an office visit with any physician on rehospitalization were similar.

A number of hospital-based programs have been developed to lower rates of rehospitalization, but few have proven successful. A recent systematic review of hospital-initiated transition programs found many of the tested alternatives had little impact on rehospitalizations. ${ }^{15}$ Exceptions were the Care Transitions Program ${ }^{16}$ and Project Re-Engineered Discharge (Project RED), ${ }^{17}$ both resource-intensive and personnelintensive approaches. A second review focused on studies of interventions to enhance handovers from hospitals to ambulatory providers found no strong evidence for any specific component. ${ }^{18}$ This finding was paralleled in a systematic review of post-discharge transition models that concluded that there is no model that is reliably effective in reducing rehospitalizations. ${ }^{19}$

Given the apparent limitations of alternatives for reducing the cost of transition efforts, there is high interest in the potential of shifting transitional care to the primary care setting, with the expectation that an office visit with a physician would be a more efficient approach. ${ }^{5}$ Several studies have assessed the potential protective effect of having an office visit after discharge using hospital-level data and analyses. Parallel studies of older Medicare enrollees discharged with heart failure and myocardial infarction found conflicting results. Both studies compared hospitals by determining the rate of claims for out-patient office visits during the 7 days after discharge and categorizing them in quartiles. ${ }^{20,21}$ For heart failure, there was an apparent threshold effect: the quartile with the lowest percent of patients with follow-up visits had more rehospitalizations during the initial 30 days post-discharge. However, for myocardial infarction patients, there was no relationship between rates of follow-up and rehospitalizations. Ecological analyses at the hospital level leave open the possibility that hospitals with higher rates of follow-up visits also have organizational structures and other characteristics that may account for lower rates of rehospitalization.

In contrast to studies of hospital rates, a third study of Medicare enrollees used individual level data to assess rehospitalization among patients with discharge diagnoses of chronic obstructive pulmonary disease. Comparisons were between office visits with the primary care physician or pulmonologist within 30 days and rehospitalization during the same period. The resulting hazard ratio of 0.91 was statistically significant. ${ }^{22}$ Other studies analyzing individual outcomes include a study tracking office visits and rehospitalization over 90 days post-discharge. An extremely protective effect was found for office visits within 7 days (odds ratio 0.10 ). ${ }^{23}$ Subjects were limited to those with no prior hospitalizations during the previous 90 days, an exclusion that is likely to produce a very different result from studies including all discharged patients. An additional study was set within a single specific hospital and tracked office visits and rehospitalizations with the same diagnosis as the index hospitalization during the initial 30 days post-discharge. A strong association was found but it was not statistically significant. ${ }^{9}$ Several single-hospital studies focused on office visits that were scheduled ${ }^{24}$ or reported in discharge summaries, ${ }^{25}$ and found small ${ }^{24}$ and no effects ${ }^{25}$ with limited ability to compare results to those studies that assessed actual office visits.

Studies focused on out-patient care for newly discharged older adults provide possible suggestions for the observed conflicting results at the hospital and patient levels. A randomized trial of intense primary care for discharged patients within the Veterans' Administration system found that patients with more primary care were more often rehospitalized, suggesting that contact with primary care physicians led to more detection of underlying problems requiring hospital-based treatment. ${ }^{26}$ A study of older adults insured by Kaiser Permanente of Northern California who were rehospitalized within 30 days of discharge included intense interviews with patients, caregivers, and primary care providers. ${ }^{27}$ Although $53 \%$ had an office visit before the rehospitalization, $65 \%$ of physicians did not know of the worsening conditions that led to the admission. Among the rehospitalizations considered preventable, interviews revealed an average of 8.7 contributing factors.

If a post-discharge follow-up visit is to succeed in reducing the risk of rehospitalization, it will need to include extensive exploration of the patient's changed medical condition, as well as provide education and support. Several guidelines have been produced, including a checklist for physicians by the California Healthcare Foundation ${ }^{28}$ and the American Medical Association's There and Home Again, Safely ${ }^{29}$ publication, which details the responsibilities of primary care practices during transitions in care. Recommended responsibilities include coordination with caregivers, case managers and home health workers, sharing of recommendations and medication instructions with all members of the team, medication reconciliation, and extensive instruction to patients and caregivers on self-management. Unfortunately, these are not tasks that 
can be readily fit within standard outpatient visits. Fulfillment of these responsibilities may necessitate a comprehensive transition program.

There are several limitations to this study. The office visit exposure could not be directly randomized, so this was an observational study with the accompanying potential for confounding. Some office visits may have been scheduled due to a patient's deteriorating health or in response to ongoing health challenges that placed the patient at high risk of rehospitalization. We drew upon the full information available from both electronic medical record and claims data to control for clear predictors of rehospitalization and also developed a high-dimensional propensity score. Models that were incrementally controlling for various levels of confounding showed only slight modifications in the hazard ratio for office visits within 7 days with either a primary care provider or any physician. However, a number of predictors of rehospitalization are not available in EHR data, such as the existence and quality of patients' support systems, the extent of patients' understanding of medication regimens and recommendations for self-management, and their ability to recognize and respond to warning signs.

Determining whether a null finding is convincing depends upon interpretation of the size of the confidence interval. Because of the limited size of the sample $(3,661$ discharges), the confidence interval is fairly wide, with a lower limit of 0.80 . Thus, the possibility of a protective effect remains. Since this study was set within one multispecialty group practice, it is important for these findings to be assessed in other settings. In order to provide evidence for decision makers considering penalties or rewards for primary care practices related to follow-up visits for discharged patients, these studies should replicate our approach by including the full range of discharged patients.

In summary, we found no protective effect for office visits within 7 days to either primary care providers or any physician. Post-discharge visits are likely to have a better chance of improving outcomes for older patients if incorporated into comprehensive transition programs.

Contributors: Author contributions: Terry S. Field, Jessica Ogarek, and Jerry H. Gurwitz contributed to conception and design, acquisition of data, analysis and interpretation of data, as well as drafting and critically revising the article for important intellectual content. George Reed contributed to conception and design, analysis and interpretation of data, and revising the article critically for important intellectual content. Lawrence Garber contributed to conception and design, acquisition of data, analysis and interpretation of data, and revising the article critically for important intellectual content. Information on author access to data: Terry S. Field and Jessica Ogarek had full access to all the data in the study and take responsibility for the integrity of the data and accuracy of the data analysis. Non-author contributions: We would like to acknowledge and thank Shawn Gagne (BA, Meyers Primary Care Institute and University of Massachusetts Medical School), Devi Sundaresan (MS, Reliant Medical Group) and Peggy Preusse (RN, Reliant Medical Group) for contributions to the collection and validation of data used in this study.
Funders: Funding source: This study was funded by grant R18 HSO17203 from the Agency for Healthcare Research and Quality (AHRQ). Role of sponsor: The funding sources had no role in the study design; in the collection, analysis, and interpretation of data; in the writing of the report; or in the decision to submit the paper for publication. Researchers were independent from the funders of the study. The content is solely the responsibility of the authors and does not necessarily represent the official views of the funders.

Conflict of Interest: The authors declare that they do not have a conflict of interest.

Corresponding Author: Terry S. Field, DSc; Meyers Primary Care Institute, A Joint Endeavor of University of Massachusetts Medical School, Reliant Medical Group, and Fallon Community Health Plan, 630 Plantation Street, Worcester, MA 01605, USA (e-mail: terry.field@umassmed.edu).

\section{REFERENCES}

1. Krumholz HM. Post-hospital syndrome-an acquired, transient condition of generalized risk. N Engl J Med. 2013;368(2):100-2.

2. Coleman EA. Falling through the cracks: challenges and opportunities for improving transitional care for persons with continuous complex care needs. J Am Geriatr Soc. 2003;51(4):549-55.

3. Forster AJ, Murff HJ, Peterson JF, Gandhi TK, Bates DW. The incidence and severity of adverse events affecting patients after discharge from the hospital. Ann Intern Med. 2003;138(3): 161-7.

4. Moore C, Wisnivesky J, Williams S, McGinn T. Medical errors related to discontinuity of care from an inpatient to an outpatient setting. J Gen Intern Med. 2003;18(8):646-51.

5. Jencks SF, Williams MV, Coleman EA. Rehospitalizations among patients in the Medicare fee-for-service program. N Engl J Med. 2009;360(14):141828.

6. Berenson RA, Paulus RA, Kalman NS. Medicare's readmissionsreduction program-a positive alternative. N Engl J Med. 2012;366(15):1364-6.

7. Joynt KE, Jha AK. Thirty-day readmissions-truth and consequences. N Engl J Med. 2012;366(15):1366-9.

8. Centers for Medicare \& Medicaid Services. Readmissions reduction program. http://www.cms.gov/Medicare/Medicare-Fee-for-Service-Payment/AcuteInpatientPPS/Readmissions-Reduction-Program.html. Last accessed October 7, 2014.

9. Misky GJ, Wald HL, Coleman EA. Post-hospitalization transitions: examining the effects of timing of primary care provider follow-up. J Hosp Med. 2010;5(7):392-7.

10. Coleman EA, Williams Mv. Executing high-quality care transitions: a call to do it right. J Hosp Med. 2007;2(5):287-90.

11. Gurwitz JH, Field TS, Ogarek J, et al. An electronic health record-based intervention to increase follow-up office visits and decrease rehospitalization in older adults. J Am Geriatr Soc. 2014;62(5):865-71.

12. Charlson ME, Pompei P, Ales KL, MacKenzie CR. A new method of classifying prognostic comorbidity in longitudinal studies: development and validation. J Chronic Dis. 1987;40(5):373-83.

13. Division of Pharmacoepidemiology \& Pharmacoeconomics, Brigham and Women's Hospital. Software Downloads: Pharmacoepidemiology Toolbox including High-dimensional Propensity Score (hd-PS) Adjustment version 2. http://www.drugepi.org/dope-downloads/\#Pharmacoepidemiology Toolbox. Last accessed October 7, 2014.

14. Schneeweiss S, Rassen JA, Glynn RJ, Avorn J, Mogun H, Brookhart MA. High-dimensional propensity score adjustment in studies of treatment effects using health care claims data. Epidemiology. 2009;20(4):512-22.

15. Rennke S, Nguyen OK, Shoeb MH, Magan Y, Wachter RM, Ranji SR. Hospital-initiated transitional care interventions as a patient safety strategy: a systematic review. Ann Intern Med. 2013;158(5 Pt 2):433-40.

16. Coleman EA, Parry C, Chalmers S, Min SJ. The care transitions intervention: results of a randomized controlled trial. Arch Intern Med. 2006; 166(17): 1822-8.

17. Jack BW, Chetty VK, Anthony D, et al. A reengineered hospital discharge program to decrease reshospitalization. Ann Intern Med. 2009;150:17887. 
18. Hesselink G, Schoonhoven L, Barach P, et al. Improving patient handovers from hospital to primary care: a systematic review. Ann Intern Med. 2012;157(6):417-28.

19. Hansen LO, Young RS, Hinami K, Leung A, Williams MV. Interventions to reduce 30-day rehospitalization: a systematic review. Ann Intern Med. 2011;155(8):520-8.

20. Hernandez AF, Greiner MA, Fonarow GC, et al. Relationship between early physician follow-up and 30-day readmission among Medicare beneficiaries hospitalized for heart failure. JAMA. 2010;303(17):171622.

21. Hess CN, Shah BR, Peng SA, Thomas L, Roe MT, Peterson ED. Association of early physician follow-up and 30-day readmission after non-ST-segment-elevation myocardial infarction among older patients. Circulation. 2013;128(11):1206-13.

22. Sharma G, Kuo YF, Freeman JL, Zhang DD, Goodwin JS. Outpatient follow-up visit and 30-day emergency department visit and readmission in patients hospitalized for chronic obstructive pulmonary disease. Arch Intern Med. 2010;170(18):1664-70.

23. Lin CY, Barnato AE, Degenholtz HB. Physician follow-up visits after acute care hospitalization for elderly Medicare beneficiaries discharged to noninstitutional settings. J Am Geriatr Soc. 2011;59(10): 1947-54.

24. Kashiwagi DT, Burton MC, Kirkland LL, Cha S, Varkey P. Do timely outpatient follow-up visits decrease hospital readmission rates? Am J Med Qual. 2012;27(1):11-5.

25. Grafft CA, McDonald FS, Ruud KL, Liesinger JT, Johnson MG, Naessens JM. Effect of hospital follow-up appointment on clinical event outcomes and mortality. Arch Intern Med. 2010;170(11):955-60.

26. Weinberger M, Oddone EZ, Henderson WG. Does increased access to primary care reduce hospital readmissions? $N$ Engl $J$ Med. 1996;334: 1441-7.

27. Feigenbaum P, Neuwirth E, Trowbridge $\mathbf{L}$, et al. Factors contributing to all-cause 30-day readmissions: a structured case series across 18 hospitals. Med Care. 2012;50(7):599-605.

28. Coleman EA. The Post-Hospital Follow-Up Visit: A Physician Checklist to Reduce Readmissions. Oakland: California HealthCare Foundation; 2010.

29. Sokol PE, Wynia MK. There and Home Again, Safely: Five Responsibilities of Ambulatory Practices in High Quality Care Transitions. Chicago: AMA Expert Panel on Care Transitions; 2013. 